
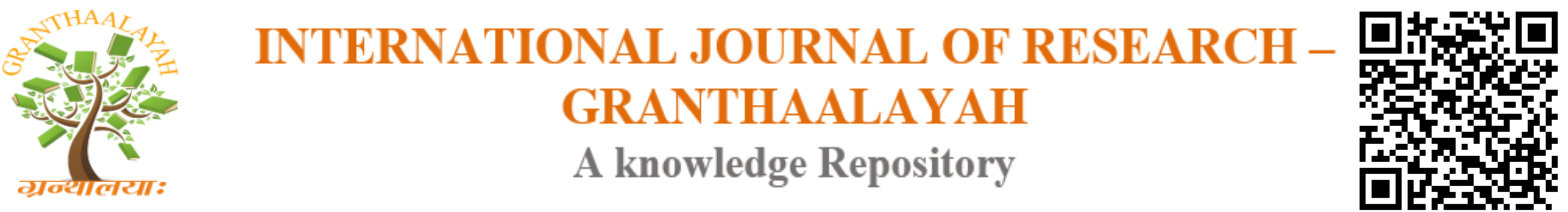

Science

\title{
ENRICHED BLACK HOLE ALGORITHM FOR DIMINUTION OF REAL POWER LOSS
}

\author{
Dr.K.Lenin *1 \\ ${ }^{* 1}$ Professor, Department of EEE, Prasad V.Potluri Siddhartha Institute of Technology, Kanuru, \\ Vijayawada, Andhra Pradesh -520007, India
}

\begin{abstract}
This paper presents an Enriched Black Hole (EBH) algorithm for solving reactive power flow problem. The Black Hole Algorithm starts with a preliminary population of contestant and for all iteration of the black hole algorithm, the most excellent candidate is favored to be the black hole, which followed by pulling further candidates around it, called stars. If a star move very close to the black hole, it will be consumed by the black hole and is vanished undyingly. In such a case, a new star - candidate solution is arbitrarily created and placed in the exploration space and starts a new search. Black hole algorithm is feeble to carry out global search completely in the large size problem spaces. So the enhancement in the amalgamation process in black hole algorithm has to be done. In this work, black hole algorithm will be enhanced, using stars gravities information. For this aim, a kind of gravitational force between stars is defined and the movement of stars to the black hole is adjusted during the penetration of solution space. In order to evaluate the projected Enriched Black Hole $(\mathrm{EBH})$ algorithm, it has been tested in Standard IEEE 57,118 bus systems and compared to other standard reported algorithms. Simulation results reveal about the Enriched performance of the projected algorithm in plummeting the real power loss.
\end{abstract}

Keywords: Optimal Reactive Power; Transmission Loss; Enriched Black Hole.

Cite This Article: Dr.K.Lenin. (2017). "ENRICHED BLACK HOLE ALGORITHM FOR DIMINUTION OF REAL POWER LOSS." International Journal of Research - Granthaalayah, 5(9), 186-194. https://doi.org/10.29121/granthaalayah.v5.i9.2017.2228.

\section{Introduction}

Optimal reactive power dispatch problem is one of the difficult optimization problems in power systems. The sources of the reactive power are the generators, synchronous condensers, capacitors, static compensators and tap changing transformers. The problem that has to be solved in a reactive power optimization is to determine the required reactive generation at various locations so as to optimize the objective function. Here the reactive power dispatch problem involves best utilization of the existing generator bus voltage magnitudes, transformer tap setting and the output of reactive power sources so as to minimize the loss and to enhance the voltage 
stability of the system. It involves a non linear optimization problem. Various mathematical techniques have been adopted to solve this optimal reactive power dispatch problem. These include the gradient method [1-2], Newton method [3] and linear programming [4-7].The gradient and Newton methods suffer from the difficulty in handling inequality constraints. To apply linear programming, the input- output function is to be expressed as a set of linear functions which may lead to loss of accuracy. Recently Global Optimization techniques such as genetic algorithms have been proposed to solve the reactive power flow problem [8, 9]. In this paper, Enriched Black Hole $(\mathrm{EBH})$ algorithm utilized for solving reactive power problem. The fundamental design of a black hole is basically an area of space that has so much mass concerted in it and there is no means for a close by object to get away from the gravitational heave. In black hole algorithm (BHA) [10-15] begins with a preliminary population of candidate solutions to an optimization problem. Black hole algorithm is feeble to carry out global search completely in the large size problem spaces. So the enhancement in the amalgamation process in black hole algorithm has to be done. In this work, black hole algorithm will be enhanced, using stars gravities information. For this aim, a kind of gravitational force between stars is defined and the movement of stars to the black hole is adjusted during the penetration of solution space. The performance of Enriched Black Hole $(\mathrm{EBH})$ algorithm has been evaluated in Standard IEEE 57,118 bus systems \& the results analysis shows that our proposed approach outperforms all approach explored in this paper.

\section{Objective Function}

\section{Active Power Loss}

Main aim of the reactive power dispatch problem is to reduce the active power loss in the transmission network, which can be described as:

$F=P L=\sum_{k \in N b r} g_{k}\left(V_{i}^{2}+V_{j}^{2}-2 V_{i} V_{j} \cos \theta_{i j}\right)$

Where $g_{k}$ : is the conductance of branch between nodes $\mathrm{i}$ and $\mathrm{j}$, Nbr: is the total number of transmission lines in power systems.

\section{Voltage Profile Improvement}

For minimization of the voltage deviation in PQ buses, the objective function turns into:

$F=P L+\omega_{v} \times V D$

Where $\omega_{\mathrm{v}}$ : is a weighting factor of voltage deviation.

VD is the voltage deviation given by:

$V D=\sum_{i=1}^{N p q}\left|V_{i}-1\right|$

\section{Equality Constraint}

The equality constraint of the Reactive power problem is represented by the power balance equation, and can be written as, where the total power generation must cover the total power demand and total power loss:

$P_{G}=P_{D}+P_{L}$

Where, $P_{G}$ - Total Power Generation, $P_{D}$-Total Power Demand, $P_{L}-$ Total Power Loss. 


\section{Inequality Constraints}

Inequality constraints define the limitations in power system components and power system security. Upper and lower bounds on the active power of slack bus, and reactive power of generators are written as follows:

$P_{\text {gslack }}^{\min } \leq P_{\text {gslack }} \leq P_{\text {gslack }}^{\text {max }}$

$Q_{g i}^{\min } \leq Q_{g i} \leq Q_{g i}^{\max }, i \in N_{g}$

Upper and lower bounds on the bus voltage magnitudes are described as follows:

$V_{i}^{\min } \leq V_{i} \leq V_{i}^{\max }, i \in N$

Upper and lower bounds on the transformers tap ratios are given as follows:

$T_{i}^{\text {min }} \leq T_{i} \leq T_{i}^{\max }, i \in N_{T}$

Upper and lower bounds on the compensators reactive powers are written as follows:

$Q_{c}^{\min } \leq Q_{c} \leq Q_{C}^{\max }, i \in N_{C}$

Where $\mathrm{N}$ is the total number of buses, $\mathrm{N}_{\mathrm{T}}$ is the total number of Transformers; $\mathrm{N}_{\mathrm{c}}$ is the total number of shunt reactive compensators.

\section{Black Hole Algorithm}

The Black Hole Algorithm (BHA) is a population-based technique that has some similar characteristics with other population-based techniques. The same as with other population-based algorithms, a population of candidate solutions to a specified problem is formed and scattered arbitrarily in the exploration space. The population-based algorithms develop the produced population in the way of the optimal solution via exact method. In the BHA algorithm the progression of the population is done by moving all the candidates in the direction of the best candidate in every iteration, which is, the black hole and replace with those candidates that come into within the range of the black hole by recently produced candidates in the exploration space. In the BHA the most outstanding candidate among all the candidates at every iteration is selected as a black hole and all the other candidates form the regular stars. The formation of the black hole is not arbitrary and it is one of the authentic candidates of the population. Then, all the candidates are stirred towards the black hole based on their existing location and an arbitrary number. Alike to other population-based algorithms, black hole algorithm (BHA) is an arbitrarily produced population of candidate solutions - the stars - are located in the exploration space of the problem. Subsequent initialization, the fitness values of the population are calculated and the most outstanding candidate in the population, which has the most outstanding fitness value, is selected to be the black hole and the rest form the regular stars. The black hole has the ability to take in the stars that surround it.

After initializing the black hole and stars, the black hole begin to take up the stars surround it and all the stars start moving in the direction of the black hole.

The amalgamation of stars by the black hole is formulated as follows:

$X_{i}(t+1)=X_{i}(t)+\operatorname{rand} \times\left(X_{B H}, X_{i}(t)\right) i=1,2, \ldots, N$ 
Where $x_{i}(t)$ and $x_{i}(t+1)$ are the locations of the ith star at iterations $t$ and $t+1$, respectively. $x_{B H}$ is the location of the black hole in the explore space. rand is a arbitrary number in the interval $[0,1] . \mathrm{N}$ is the number of stars (candidate solutions).

While moving in the direction of the black hole, a star might reach a position with lower cost than the black hole. In such a case, the black hole moves about to the position of that star and vice versa. Then the BHA algorithm will go on with the black hole in the fresh location and then stars start moving in the direction of this fresh location. In addition, there is a possibility of crossing the event horizon at some stage of moving stars towards the black hole. Every star (candidate solution) that crosses the event horizon of the black hole will be sucked by the black hole. Every time a candidate (star) expire - it is sucked in by the black hole - an additional candidate solution (star) is born and dispersed arbitrarily in the explore space and starts a fresh search. This is done to maintain the number of candidate solutions constant. Subsequent iteration takes place after all the stars have been moved.

The radius of the event horizon in the black hole algorithm is computed using the following equation:

$R=\frac{f_{B H}}{\sum_{i=1}^{N} f_{i}}$

Where $f_{\mathrm{BH}}$ is the fitness value of the black hole and fi is the fitness value of the ith star. $\mathrm{N}$ is the number of stars (candidate solutions). When the distance between a candidate solution and the black hole (best candidate) is less than R, that candidate is collapsed and a new candidate is created and distributed randomly in the search space. Based on the above explanation the key steps in the BHA algorithm are concise as follows:

a) Initialize a population of stars with arbitrary locations in the explore space Loop

b) For every star, calculate the objective function

c) Pick the most excellent star that has the most excellent fitness value as the black hole

d) Modify the position of every star according to Eq. (10)

e) If a star attains a position with lower cost than the black hole, then swap their locations

f) If a star crosses the event horizon of the black hole, substitute it with a fresh star in an arbitrary location in the search space

g) If a stop criterion is met, exit the loop End loop

\section{Enriched Black Hole Algorithm (EBH)}

Black hole algorithm is feeble to carry out global search completely in the large size problem spaces. So the enhancement in the amalgamation process in black hole algorithm has to be done. Two significant characteristics of the swarm-based methods are exploration and exploitation. The exploration is related to penetrating of space, where the exploitation is search for the optimum. The exploration is a significant theme in swarm-based heuristic algorithms. Over time, exploring will be condensed and exploitation aptitude lightens in, so the algorithm alters itself in the semi-optimal points. There should be equilibrium between exploration and exploitation, to keep black hole algorithm protected from trapping in local optima. In this work, black hole 
algorithm will be enhanced, using stars gravities information. For this aim, a kind of gravitational force between stars is defined and the movement of stars to the black hole is adjusted during the penetration of solution space.

There will be Swarm with N stars. The location of the ith stars $\left(\mathrm{X}_{\mathrm{i}}\right)$ is defined by Eq. (12). $X_{i}=\left(\operatorname{star}_{i}, \ldots\right.$, star $_{N}$, blcakhole $\left._{d}\right)$

Where star $_{\mathrm{i}}$ is the position of ith star and blackhole $\mathrm{d}_{\mathrm{d}}$ is the position of $\mathrm{d}^{\text {th }}$ black hole, respectively.

At a exact time " $t$ ", we define the amalgamation acting on star "i" from star " $\mathrm{j}$ " as Eq. (13).

$E_{i j}^{d}=\xi\left(t_{o}\right) \frac{C_{p i}(t) \times C_{a j}(t) \times\left(\operatorname{star}_{j}(t)-\operatorname{star}_{i}(t)\right)}{\left(D_{i j}(t)+\varepsilon\right)^{2} \times\left(C_{p i}(t)+C_{a j}(t)\right)} \times\left(\frac{t_{o}}{t-t_{o}}\right)^{a}$

Where $\mathrm{C}_{\mathrm{aj}}$ is the power of star $\mathrm{j}, \mathrm{C}_{\mathrm{pi}}$ is the power associated to star $\mathrm{i}, \xi\left(\mathrm{t}_{0}\right)$ is preliminary incorporation constant, $\varepsilon$ is a small constant, and $D_{i j}(t)$ is distance between two stars $i$ and $j$. To give a stochastic characteristic to black hole algorithm, entire force is arbitrarily weighted sum of the forces of others (Eq. (14)).

$E_{i}^{d}(t)=\sum_{j=1 J \neq i}^{N} \operatorname{random}_{i} E_{i j}^{d}(t)$

Where $\operatorname{random}_{j}$ is in $[0,1]$. Hence, the acceleration of the star $i$ at time $t$, and in direction $d^{\text {th }}$, is given by Eq. (15).

$a_{i}^{d}(t)=\frac{E_{i}^{d}(t)}{C_{i i}(t)}$

Where $C_{i i}$ is the Power of $i^{\text {th }}$ star, the next velocity of star is considered as follows. Consequently, position and its velocity are designed based on Eq. (16) and Eq. (17).

$v_{i}^{d}(t+1)=$ random $_{i} \times v_{i}^{d}(t)+a_{i}^{d}(t)$

$\operatorname{star}_{i}(t+1)=\operatorname{star}_{i}(t)+v_{i}^{d}(t+1)$

Where random $_{i}$ is in $[0,1]$. This arbitrary number is for randomization of the exploration.

\section{Simulation Results}

At first Enriched Black Hole (EBH) algorithm has been tested in standard IEEE-57 bus power system. The reactive power compensation buses are 18, 25 and 53. Bus 2, 3, 6, 8, 9 and 12 are PV buses and bus 1 is selected as slack-bus. The system variable limits are given in Table 1.

The preliminary conditions for the IEEE-57 bus power system are given as follows:

$\mathrm{P}_{\text {load }}=12.128$ p.u. $\mathrm{Q}_{\text {load }}=3.062$ p.u.

The total initial generations and power losses are obtained as follows:

$\sum P_{G}=12.472$ p.u. $\sum Q_{G}=3.3164$ p.u.

$\mathrm{P}_{\text {loss }}=0.25872$ p.u. $\mathrm{Q}_{\text {loss }}=-1.2074$ p.u.

Table 2 shows the various system control variables i.e. generator bus voltages, shunt capacitances and transformer tap settings obtained after optimization which are within the 
acceptable limits. In Table 3, shows the comparison of optimum results obtained from proposed methods with other optimization techniques. These results indicate the robustness of proposed approaches for providing better optimal solution in case of IEEE-57 bus system.

Table 1: Variable Limits

\begin{tabular}{|c|c|c|c|c|c|c|c|}
\hline \multicolumn{8}{|c|}{ Reactive Power Generation Limits } \\
\hline Bus no & 1 & 2 & 3 & 6 & 8 & 9 & 12 \\
\hline Qgmin & -1.4 & -.015 & -.02 & -0.04 & -1.3 & -0.03 & -0.4 \\
\hline Qgmax & 1 & 0.3 & 0.4 & 0.21 & \multicolumn{2}{|c|}{0.04} & 1.50 \\
\hline \multicolumn{8}{|c|}{ Voltage And Tap Setting Limits } \\
\hline vgmin & Vgmax & \multicolumn{2}{|c|}{ vpqmin } & \multicolumn{2}{|c|}{ Vpqmax } & tkmin & tkmax \\
\hline 0.9 & 1.0 & 0.9 & & 1.05 & & \begin{tabular}{|l}
0.9 \\
\end{tabular} & 1.0 \\
\hline \multicolumn{5}{|c|}{ Shunt Capacitor Limits } & & & \\
\hline \multirow{2}{*}{$\begin{array}{l}\text { Bus no } \\
\text { Qcmin }\end{array}$} & 18 & \multicolumn{2}{|c|}{25} & \multicolumn{2}{|l|}{53} & & \\
\hline & \multicolumn{2}{|l|}{0} & 0 & \multicolumn{2}{|l|}{0} & & \\
\hline Qcmax & 10 & \multicolumn{2}{|c|}{5.2} & \multicolumn{2}{|l|}{6.1} & & \\
\hline
\end{tabular}

Table 2: Control variables obtained after optimization

\begin{tabular}{|l|l|}
\hline Control Variables & EBH \\
\hline V1 & 1.1 \\
\hline V2 & 1.032 \\
\hline V3 & 1.030 \\
\hline V6 & 1.021 \\
\hline V8 & 1.022 \\
\hline V9 & 1.009 \\
\hline V12 & 1.014 \\
\hline Qc18 & 0.0661 \\
\hline Qc25 & 0.200 \\
\hline Qc53 & 0.0470 \\
\hline T4-18 & 1.009 \\
\hline T21-20 & 1.044 \\
\hline T24-25 & 0.862 \\
\hline T24-26 & 0.872 \\
\hline T7-29 & 1.052 \\
\hline T34-32 & 0.874 \\
\hline T11-41 & 1.013 \\
\hline T15-45 & 1.030 \\
\hline T14-46 & 0.910 \\
\hline T10-51 & 1.020 \\
\hline T13-49 & 1.060 \\
\hline T11-43 & 0.910 \\
\hline T40-56 & 0.900 \\
\hline T39-57 & 0.950 \\
\hline T9-55 & 0.950 \\
\hline
\end{tabular}


Table 3: Comparison results

\begin{tabular}{|l|l|l|l|l|}
\hline S.No. & Optimization Algorithm & Finest Solution & Poorest Solution & Normal Solution \\
\hline 1 & NLP [16] & 0.25902 & 0.30854 & 0.27858 \\
\hline 2 & CGA [16] & 0.25244 & 0.27507 & 0.26293 \\
\hline 3 & AGA [16] & 0.24564 & 0.26671 & 0.25127 \\
\hline 4 & PSO-w [16] & 0.24270 & 0.26152 & 0.24725 \\
\hline 5 & PSO-cf [16] & 0.24280 & 0.26032 & 0.24698 \\
\hline 6 & CLPSO [16] & 0.24515 & 0.24780 & 0.24673 \\
\hline 7 & SPSO-07 [16] & 0.24430 & 0.25457 & 0.24752 \\
\hline 8 & L-DE [16] & 0.27812 & 0.41909 & 0.33177 \\
\hline 9 & L-SACP-DE [16] & 0.27915 & 0.36978 & 0.31032 \\
\hline 10 & L-SaDE [16] & 0.24267 & 0.24391 & 0.24311 \\
\hline 11 & SOA [16] & 0.24265 & 0.24280 & 0.24270 \\
\hline 12 & LM [17] & 0.2484 & 0.2922 & 0.2641 \\
\hline 13 & MBEP1 [17] & 0.2474 & 0.2848 & 0.2643 \\
\hline 14 & MBEP2 [17] & 0.2482 & 0.283 & 0.2592 \\
\hline 15 & BES100 [17] & 0.2438 & 0.263 & 0.2541 \\
\hline 16 & BES200 [17] & 0.3417 & 0.2486 & 0.2443 \\
\hline 17 & Proposed EBH & 0.22012 & 0.23008 & 0.22214 \\
\hline
\end{tabular}

Then Enriched Black Hole (EBH) algorithm has been tested in standard IEEE 118-bus test system [18]. The system has 54 generator buses, 64 load buses, 186 branches and 9 of them are with the tap setting transformers. The limits of voltage on generator buses are $0.95-1.1$ per-unit., and on load buses are $0.95-1.05$ per-unit. The limit of transformer rate is $0.9-1.1$, with the changes step of 0.025 . The limitations of reactive power source are listed in Table 4 , with the change in step of 0.01 .

Table 4: Limitation of reactive power sources

\begin{tabular}{|l|l|l|l|l|l|l|l|}
\hline BUS & 5 & 34 & 37 & 44 & 45 & 46 & 48 \\
\hline QCMAX & 0 & 14 & 0 & 10 & 10 & 10 & 15 \\
\hline QCMIN & -40 & 0 & -25 & 0 & 0 & 0 & 0 \\
\hline BUS & 74 & 79 & 82 & 83 & 105 & 107 & 110 \\
\hline QCMAX & 12 & 20 & 20 & 10 & 20 & 6 & 6 \\
\hline QCMIN & 0 & 0 & 0 & 0 & 0 & 0 & 0 \\
\hline
\end{tabular}

The statistical comparison results of 50 trial runs have been list in Table 5 and the results clearly show the better performance of proposed Enriched Black Hole $(\mathrm{EBH})$ algorithm in reducing the real power loss.

Table 5: Comparison results

\begin{tabular}{|l|l|l|l|l|}
\hline Active power loss (MW) & $\begin{array}{l}\text { BBO } \\
{[\mathbf{1 9 ]}}\end{array}$ & $\begin{array}{l}\text { ILSBBO/ } \\
\text { strategy1 [19] }\end{array}$ & $\begin{array}{l}\text { ILSBBO/ } \\
\text { strategy1 [19] }\end{array}$ & $\begin{array}{l}\text { Proposed } \\
\text { EBH }\end{array}$ \\
\hline Min & 128.77 & 126.98 & 124.78 & 117.02 \\
\hline Max & 132.64 & 137.34 & 132.39 & 119.88 \\
\hline Average & 130.21 & 130.37 & 129.22 & 118.94 \\
\hline
\end{tabular}




\section{Conclusion}

In this paper a novel approach Enriched Black Hole (EBH) algorithm used to solve reactive power problem, considering various generator constraints, has been successfully applied.The performance of the proposed Enriched Black Hole (EBH) algorithm has been has been tested in standard IEEE 57,118 bus systems and simuation results represent about the reduction of real power loss when compared with other standard reported algorithms and generally volatge profiles are within the limits.

\section{References}

[1] O.Alsac,and B. Scott, "Optimal load flow with steady state security”,IEEE Transaction. PAS 1973, pp. 745-751.

[2] Lee K Y ,Paru Y M , Oritz J L -A united approach to optimal real and reactive power dispatch , IEEE Transactions on power Apparatus and systems 1985: PAS-104 : 1147-1153

[3] A.Monticelli , M .V.F Pereira, and S. Granville, "Security constrained optimal power flow with post contingency corrective rescheduling", IEEE Transactions on Power Systems :PWRS-2, No. 1, pp.175-182.,1987.

[4] Deeb N ,Shahidehpur S.M ,Linear reactive power optimization in a large power network using the decomposition approach. IEEE Transactions on power system 1990: 5(2) : 428-435

[5] E. Hobson ,'Network consrained reactive power control using linear programming, ' IEEE Transactions on power systems PAS -99 (4) ,pp 868=877, 1980

[6] K.Y Lee ,Y.M Park, and J.L Oritz, "Fuel -cost optimization for both real and reactive power dispatches", IEE Proc; 131C,(3), pp.85-93.

[7] M.K. Mangoli, and K.Y. Lee, "Optimal real and reactive power control using linear programming”, Electr.Power Syst.Res, Vol.26, pp.1-10,1993.

[8] K.Anburaja, "Optimal power flow using refined genetic algorithm", Electr.Power Compon.Syst , Vol. 30, 1055-1063,2002.

[9] D. Devaraj, and B. Yeganarayana, "Genetic algorithm based optimal power flow for security enhancement", IEE proc-Generation.Transmission and. Distribution; 152, 6 November 2005.

[10] Abdolreza Hatamlou "Black hole: A new heuristic optimization approach for data clustering", Information Sciences 222 (2013) 175-184.

[11] L. Kaper, E. Heuvel, P. Woudt, R. Giacconi, "Black hole research past and future, in: Black Holes in Binaries and Galactic Nuclei: Diagnostics, Demography and Formation", Springer, Berlin/Heidelberg, 2001, pp. 3-15.

[12] C. Pickover, "Black Holes: A Traveler's Guid”e, John Wiley \& Sons, 1998.

[13] J. Zhang, K. Liu, Y. Tan, X. He, "Random black hole particle swarm optimization and its application", in: 2008 IEEE International Conference Neural Networks and Signal Processing, ICNNSP, 2008, pp. 359-365.

[14] A. A. Heidari * , R. A. Abbaspour, "Improved Black Hole Algorithm for Efficient Low Observable UCAV Path Planning in Constrained Aerospace", ACSIJ Advances in Computer Science: an International Journal, Vol. 3, Issue 3, No.9, May 2014 ISSN : 2322-5157.

[15] Hatamlou, A., "Black hole: A new heuristic optimization approach for data clustering", Information Sciences 222, 2013, pp.175-184.

[16] Chaohua Dai, Weirong Chen, Yunfang Zhu, and Xuexia Zhang, "Seeker optimization algorithm for optimal reactive power dispatch,” IEEE Trans. Power Systems, Vol. 24, No. 3, August 2009, pp. 1218-1231.

[17] J. R. Gomes and 0. R. Saavedra, "Optimal reactive power dispatch using evolutionary computation: Extended algorithms," IEE Proc.-Gener. Transm. Distrib.. Vol. 146, No. 6. Nov. 1999. 
[18] IEEE, "The IEEE 30-bus test system and the IEEE 118-test system", (1993), http://www.ee.washington.edu/trsearch/pstca/.

[19] Jiangtao Cao, Fuli Wang and Ping Li, "An Improved Biogeography-based Optimization Algorithm for Optimal Reactive Power Flow” International Journal of Control and Automation Vol.7, No.3 (2014), pp.161-176.

*Corresponding author.

E-mail address: gklenin@ gmail.com 